\title{
Temperature Gradient Controlled Crystal Growth from TIPS Pentacene-Poly( $\alpha$-methyl styrene) Blends for Improving Performance of Organic Thin Film Transistors
}

Kyeiwaa Asare-Yeboah, Sheng Bi, Zhengran He, and Dawen Li*

*Prof. Dawen Li, Kyeiwaa Asare-Yeboah, Sheng Bi, and Dr. Zhengran He Department of Electrical and Computer Engineering

Center for Materials for Information Technology

The University of Alabama

Tuscaloosa, AL 35487, USA

* Corresponding author: Prof. Dawen Li

Email: dawenl@eng.ua.edu

Phone number: (205)348-9930

Fax number: (205)348-6959

Keywords: (Crystal growth, temperature gradient, TIPS pentacene, poly( $\alpha$-methyl styrene), organic thin-film transistors) 


\begin{abstract}
6,13-bis(triisopropylsilylethynyl) pentacene (TIPS pentacene) from simple drop casting typically forms crystals with random orientation and poor areal coverage, which leads to device-to-device performance variation of organic thin film transistors (OTFTs). Previously, a temperature gradient technique was developed to address these problems. However, this approach simultaneously introduced thermal cracks due to the thermally induced stress during crystallization. These thermal cracks accounted for a reduction of charge transport, thereby impacting the device performance of TIPS pentacene based OTFTs. In this work, an insulating polymer, poly $(\alpha$-methyl styrene) (PaMS) was blended with TIPS pentacene to relieve the thermal stress and effectively prevent the generation of thermal cracks. The results demonstrate that the incorporation of $\mathrm{P} \alpha \mathrm{MS}$ polymer combined with the temperature gradient technique improves both the hole mobility and performance consistency of TIPS pentacene based OTFTs.
\end{abstract}




\section{Introduction}

The performance of organic thin-film transistors (OTFTs) based on solution-grown organic small-molecule semiconductors, such as 6,13-bis(triisopropylsilylethynyl) pentacene (TIPS pentacene) [1-3], 5,11-bis(triethylsilylethynyl) anthradithiophene (TES-ADT) [4-6], and 2,7dioctyl[1]benzothieno[3,2-b][1]benzothiophene (C8-BTBT) [7-9], has notably advanced in recent years. The enhancement in OTFT performance situates these small-molecule semiconductors at the forefront as promising candidates for the next-generation large-area flexible electronics [1012]. Nevertheless, maintaining device-to-device performance uniformity of these small-molecule based OTFTs still remains a challenge $[13,14]$ because of the non-uniform crystal orientation in the active layer $[15,16]$. Recently, we developed a simple yet unique solution processing method, the temperature gradient technique, to control crystal growth of pure TIPS pentacene semiconductor [17]. With such an approach, the intrinsic crystal misalignment is reduced, and the resulting film exhibits large crystal sizes and substantial areal coverage. The temperature gradient technique, however, simultaneously introduces thermal cracks, which impact charge transport and reduce the hole mobility of TIPS pentacene OTFTs $[18,19]$. Therefore, it is mandatory to eliminate the thermal cracks in the TIPS pentacene films in order to realize highperformance OTFTs.

The long chains of polymers are anticipated to provide a flexible matrix to the TIPS pentacene crystals which could relieve the thermal stress, thereby preventing the occurrence of thermal cracks associated with the temperature gradient technique. In addition, various polymer additives, particularly poly( $\alpha$-methyl styrene) (P $\alpha \mathrm{MS})$ [20], have demonstrated a vertical phase segregation within the TIPS pentacene active layer, improving charge transport [21, 22]. Therefore, it is expected that incorporating P $\alpha$ MS into TIPS pentacene could result in an 
enhancement of OTFT performance by preventing thermal cracks and utilizing its capability to increase charge transport of TIPS pentacene film [23, 24].

In this study, we demonstrate that incorporation of insulating polymer, P $\alpha \mathrm{MS}$, into TIPS pentacene is able to effectively prevent the generation of thermal cracks and further enhance crystal alignment in the temperature gradient approach. As a result, the average mobility and device performance consistency have been improved. This crystal growth method which applies temperature gradient to a TIPS pentacne/P $\alpha$ MS blend is also employed to fabricate TIPS pentacene OTFTs on ITO/polyethylene terephthalate (PET) flexible substrate with poly(4vinylphenol) (PVP) as the gate dielectric. Maximum hole mobilities of $0.25 \mathrm{~cm}^{2} / \mathrm{Vs}$ and 0.5 $\mathrm{cm}^{2} /$ Vs were achieved from TIPS pentacene/PaMS OTFTs on $\mathrm{SiO}_{2} / \mathrm{Si}$ and ITO/PET flexible substrates, respectively.

\section{Experiment}

TIPS pentacene, PVP (molecular weight $\mathrm{M}_{\mathrm{w}}=25 \mathrm{k}$ ) and toluene (anhydrous, 99.8\%) were purchased from Sigma Aldrich. PaMS $\left(\mathrm{M}_{\mathrm{w}}=108 \mathrm{k}\right)$ with a polydispersity index of 1.02 was purchased from Polymer Standards Service. All materials were used as received without further purification. A temperature gradient was established by heating one side of a petri dish while the other side was kept in ambient air for 30 minutes in order to reach thermal stability. A heavily doped $n$-type silicon substrate with a $300 \mathrm{~nm}$ thickness of $\mathrm{SiO}_{2}$ layer was positioned on the petri dish in the established temperature gradient region for an additional 15 minutes to reach the desired temperature. The temperatures were measured using a type-K thermocouple beaded probe. 
To form an active layer, TIPS pentacene/P $\alpha$ MS blends in toluene $(5 \mathrm{mg} / \mathrm{mL})$ were drop casted onto the substrate inside the petri dish. The samples were then sealed inside the petri dish with a few layers of parafilm, allowing crystallization in a solvent rich environment. The slow solvent evaporation facilitates crystal growth, leading to large crystal sizes. The temperature gradient employed for crystal growth was around $3{ }^{\circ} \mathrm{C} / \mathrm{cm}-5.5^{\circ} \mathrm{C} / \mathrm{cm}$. Optical micrographs of TIPS pentacene/P $\alpha$ MS blend films were taken using a Zeiss Axioplan optical microscope with a built-in camera, and the film topography was characterized using Vecco Dektak Series V profilometer. After the formation of the TIPS pentacene active layer, $50 \mathrm{~nm}$ gold ( $\mathrm{Au}$ ) was deposited through a shadow mask via thermal evaporation as the OTFT source and drain contacts. In addition, TIPS pentacene/PaMS OTFTs were also fabricated on flexible ITO/PET substrates with a PVP polymer layer as gate dielectric. To form gate dielectric layer, PVP and a crosslinking agent, poly(melamine-co-formaldehyde) methylated solution (PMF), were first mixed (1:1 weight ratio) in propylene glycol monomethyl ether acetate (PGMEA $\geq 99.5 \%)$ at a concentration of $8 \mathrm{wt} \%$, and stirred for 24 hours. After the PVP-PMF in PGMEA solution was spin coated onto clean ITO/PET substrates at $2000 \mathrm{rpm}$ for 60 seconds, the substrates were cured at $180^{\circ} \mathrm{C}$ in a vacuum oven for 30 minutes to generate thermal cross-linking in the PVP layer. The thickness of the PVP-PMF dielectric layer was measured to be $280 \mathrm{~nm}$ with cross-sectional SEM imaging (JOEL 7000 Scanning Electron Microscope). As the final step, Au was deposited on the ITO/PET flexible substrates to complete the OTFT fabrication. The thickness of the source and drain electrodes was $100 \mathrm{~nm}$ to accommodate the active layer's large surface roughness.

Electrical characterization of OTFTs was performed in an ambient environment at room temperature using an Agilent B1500A semiconductor parameter analyzer. Field-effect hole 
mobility in the saturation regime was extracted from the slope of the transfer characteristic $\left(\mathrm{IDS}^{1 / 2}-\mathrm{V}_{\mathrm{GS}}\right.$, based on the traditional MOSFET equation:

$$
I_{D S}=\mu C_{i} \frac{W}{2 L}\left(V_{G S}-V_{T}\right)^{2}
$$

where $\mu$ is the saturation mobility, $C_{i}$ is the capacitance of the gate dielectric per unit area, $W$ and $L$ are the OTFT channel width and length, respectively, and $V_{T}$ is the threshold voltage.

All devices were measured three times to ensure the consistency of the extracted mobilities. The capacitance of the $300 \mathrm{~nm}$ silicon dioxide is $11.50 \mathrm{nF} / \mathrm{cm}^{2}$. The capacitance of PVP dielectric layer was extracted from capacitance-voltage measurement to be $10.75 \mathrm{nF} / \mathrm{cm}^{2}$.

\section{Results and Discussion}

Figure 1 illustrates how the temperature gradient method works to effectively align the TIPS pentacene crystals. The temperature gradient leads to a solubility difference within the solution, which results in a higher density of nucleation seeds in the lower temperature region. The TIPS pentacene crystal growth commences from the lower temperature region towards the higher temperature region when the solution reaches supersaturation, as shown in Figures 1(a-c). With the incorporation of PaMS polymer, thermal cracking that could have developed in the crystalline film is eliminated and the uniformity of crystal orientation is further improved. The well-aligned crystals, enhanced areal coverage and film uniformity are achieved by the combination of the temperature gradient technique and the addition of the PaMS polymer, which is illustrated in the cartoon of Figure 1(d) and also demonstrated over the entire substrate in the digital image of Figure 1(e). 
Figures 2(a-c) show the morphologies of pure TIPS pentacene films with the application of the temperature gradient technique. Employing a temperature gradient overcomes the random orientation of crystals and poor areal coverage as compared to those films from simple drop casting [12, 17, 25]; however, temperature gradient method also causes the development of undesired thermal cracks in the film as observed in these images. Figures $2(\mathrm{~d}-\mathrm{f})$ show the morphologies of the TIPS pentacene films with the addition of P $\alpha$ MS polymer, in which no thermal cracks are generated. As noticed, the crystal width is smaller when compared to those without the addition of PaMS polymer. It is anticipated that the reduced crystal width, along with PaMS polymer matrix within the film, effectively relieves the thermally induced stress and thereby prevents the generation of thermal cracks during crystallization. Among the TIPS pentacene/P $\alpha$ MS films, three different TIPS pentacene to PaMS blending ratios, 1:1, 2:1 and 3:1, were examined, but no major differences in morphology in terms of crystal size, areal coverage and crystal orientation were observed. It is worth mentioning that the stripes between the welloriented TIPS pentacene crystals in these images result from TIPS pentacene/P $\alpha$ MS molecules that were not fully crystallized but deposited onto the substrate. The stripe regions of the film are more amorphous rather than crystalline. The film topography shown in Figure 3 demonstrates a more uniform surface profile of the TIPS pentacene/P $\alpha \mathrm{MS}$ film compared with that of pure TIPS pentacene from simple drop casting; thereby indicating an improvement in the surface smoothness and uniformity from the polymer/small-molecule blend with the temperature gradient method.

Bottom-gate, top-contact OTFTs were fabricated to test charge transport in the TIPS pentacene/P $\alpha \mathrm{MS}$ blend film. Figures 4(a) and 4(b) show the transfer and output curves of the TIPS pentacene/P $\alpha \mathrm{MS}$ based OTFTs obtained from the optimal blend ratio of $2: 1$ at a $3.5^{\circ} \mathrm{C} / \mathrm{cm}$ 
temperature gradient, respectively. The slight nonlinearlity in the subthreshold region of the output curves indicate large contact resistance of drop cast TIPS pentacene OTFTs with a top contact, bottom gate structure [26]. A threshold voltage $\mathrm{V}_{\mathrm{T}}$ around $5 \mathrm{~V}$, current on/off ratio greater than $5 \times 10^{4}$, and subthreshold swing of $2.6 \mathrm{~V} / \mathrm{dec}$ were extracted from the transfer characteristics.

To demonstrate the potential of the solution processed TIPS pentacene/P $\alpha \mathrm{MS}$ film via the temperature gradient approach for flexible device applications, OTFTs were also fabricated on flexible ITO/PET substrates. Cross-linked PVP-PMF was used as the gate dielectric because it is chemically inert against the toluene solvent used to dissolve the TIPS pentacene/P $\alpha$ MS blends, and PVP has a well matched surface energy with TIPS pentacene [27], thereby promoting the adhesion of the hydrophobic silyl groups of TIPS pentacene to the substrate. Figure 5(a) shows the device configuration of bottom-gate, top-contact TIPS pentacene/P $\alpha$ MS OTFTs with PVPPMF dielectric on the ITO/PET substrate. The corresponding digital image of patterned OTFTs is presented in Figure 5(b). Figure 5(c) and 5(d) show the transfer and output characteristics acquired from the best TIPS pentacene/P $\alpha$ MS OTFTs on ITO/PET substrates, respectively. A threshold voltage $\mathrm{V}_{\mathrm{T}}$ around $-4 \mathrm{~V}$, current on/off ratio greater than $10^{3}$, and subthreshold swing of $3.3 \mathrm{~V} /$ dec were obtained.

In order to extract the mobility, parallel plate capacitors (ITO- (PVP-PMF) -Au) were fabricated and capacitance-voltage measurements were performed to determine the capacitance. The capacitance per unit area measured in accumulation regime with a bias voltage of - $20 \mathrm{~V}$ was attained as $10.75 \mathrm{nF} / \mathrm{cm}^{2}$. The average mobility along with the standard deviation of mobility was calculated to be $0.37 \pm 0.12 \mathrm{~cm}^{2} / \mathrm{Vs}$ from the measurement of five OTFTs. The highest fieldeffect hole mobility was extracted to be $0.5 \mathrm{~cm}^{2} / \mathrm{Vs}$. For comparison, a maximum average 
mobility of $0.18 \pm 0.07 \mathrm{~cm}^{2} / \mathrm{Vs}$ and the highest hole mobility of $0.25 \mathrm{~cm}^{2} / \mathrm{Vs}$ were obtained from TIPS pentacene/PaMS OTFTs on silicon substrate. The higher mobility on ITO/PET flexible substrate is attributed to the hydrophobic nature of PVP-PMF dielectric surface, which promotes the adhesion of silyl group from TIPS pentacene and molecular stacking [28]. Compared with an average mobility of $0.06 \pm 0.05 \mathrm{~cm}^{2} / \mathrm{Vs}$ from pure TIPS pentacene via temperature gradient method only, the mobility increased from the order of magnitude of $10^{-2} \mathrm{~cm}^{2} / \mathrm{Vs}$ [17] to about $10^{-}$ ${ }^{1} \mathrm{~cm}^{2} / \mathrm{Vs}$ for the TIPS pentacene/P $\alpha \mathrm{MS}$ blend based OTFTs in this work. The increase in mobility is mainly attributed to the improvement of crystal orientation and elimination of the thermal cracks. The average mobilities are summarized in Figure 6(a). It is worth mentioning that, although hexamethyldisilazane (HMDS) treatment has been employed in most literature works to passivate the silanol groups on the silicon dioxide surface to enhance charge transport [25], this treatment was not utilized in this study since the TIPS pentacene/P $\alpha$ MS solution suffered from severe dewetting on the HMDS treated surface, which considerably reduced the film areal coverage.

In addition, the performance consistency, which is defined as the ratio of the average mobility $\left(\mu_{\text {Avg }}\right)$ to the standard deviation of mobility $\left(\mu_{\text {Stdev }}\right)$, was also calculated. As shown in Figure 6(b), the pure TIPS pentacene based OTFTs exhibit $\mu_{\text {Avg }} / \mu_{\text {Stdev }}$ of 1.2, whereas TIPS pentacene/PaMS OTFTs on silicon substrate and ITO/PET flexible substrate show $\mu_{\text {Avg }} / \mu_{\text {Stdev }}$ of 2.6 and 3.1, respectively, indicating effective improvement of OTFT performance consistency.

\section{Conclusions}


In summary, we demonstrated that employing a temperature gradient technique coupled with the addition of a P $\alpha \mathrm{MS}$ insulating polymer not only significantly improves the crystal alignment and enhances areal coverage of the TIPS pentacene film, but also concurrently eliminates the thermal cracks introduced by the application of temperature gradient. The elimination of thermal cracks promotes charge transport and increases mobility from the order of magnitude of $10^{-2} \mathrm{~cm}^{2} / \mathrm{Vs}$ via temperature gradient technique only to about $10^{-1} \mathrm{~cm}^{2} / \mathrm{Vs}$, in this work. The effectiveness and versatility of the temperature gradient method with the incorporation of PaMS polymer was also demonstrated on a flexible ITO/PET substrate, and a hole mobility of $0.5 \mathrm{~cm}^{2} / \mathrm{Vs}$ was obtained.

\section{Acknowledgments}

This work was supported by National Science Foundation ECCS-1151140 and EPS-1158862. 

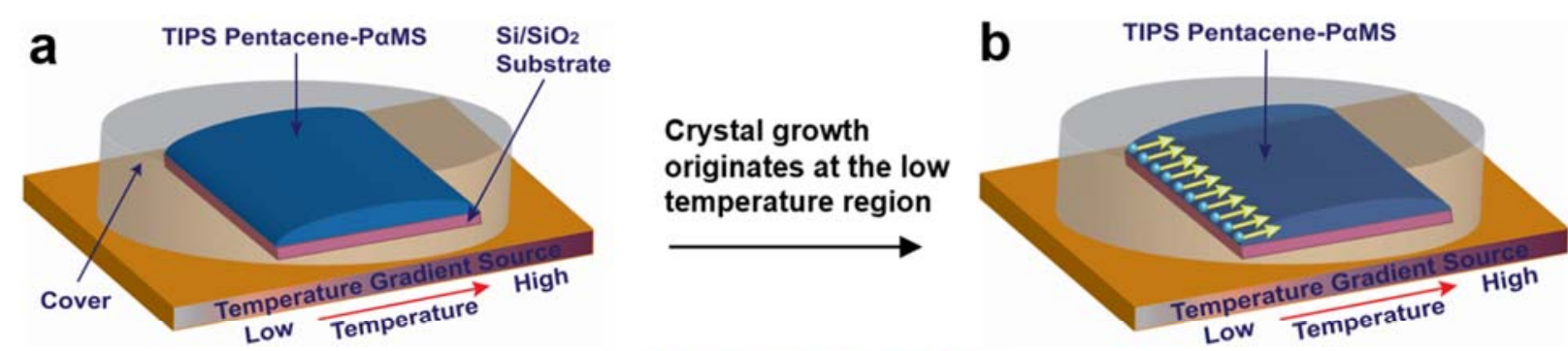

e

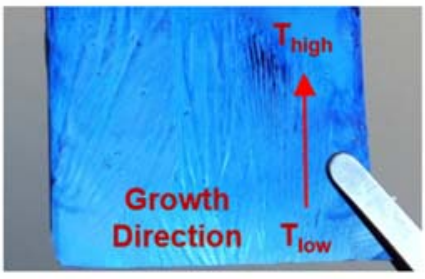

\section{Crystal growth} continues towards the high temperature end
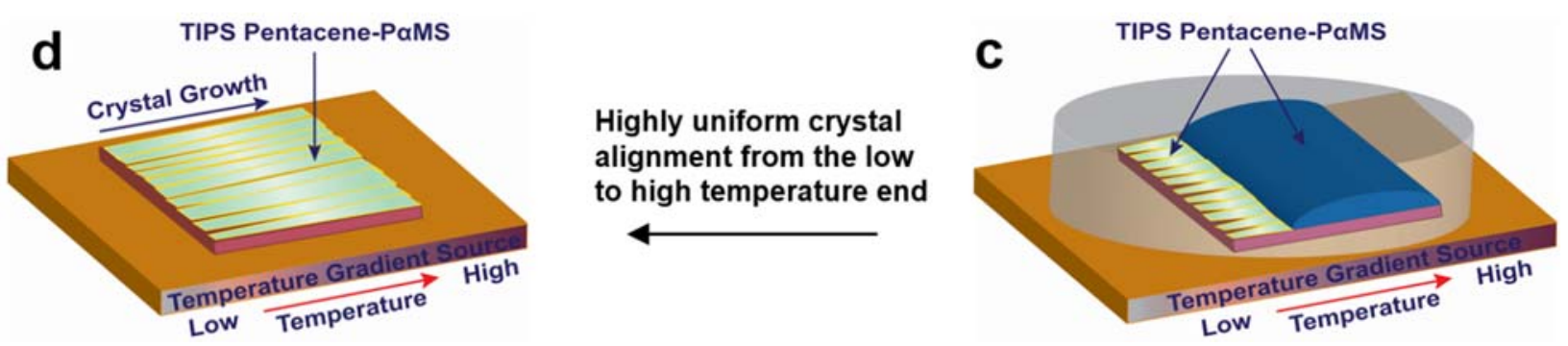

Figure 1. (a-d) Schematic of crystal growth using the temperature gradient technique. (e) A digital image of the resultant TIPS pentacene crystals from the combination of temperature gradient technique and addition of $\mathrm{P} \alpha \mathrm{MS}$ polymer. 


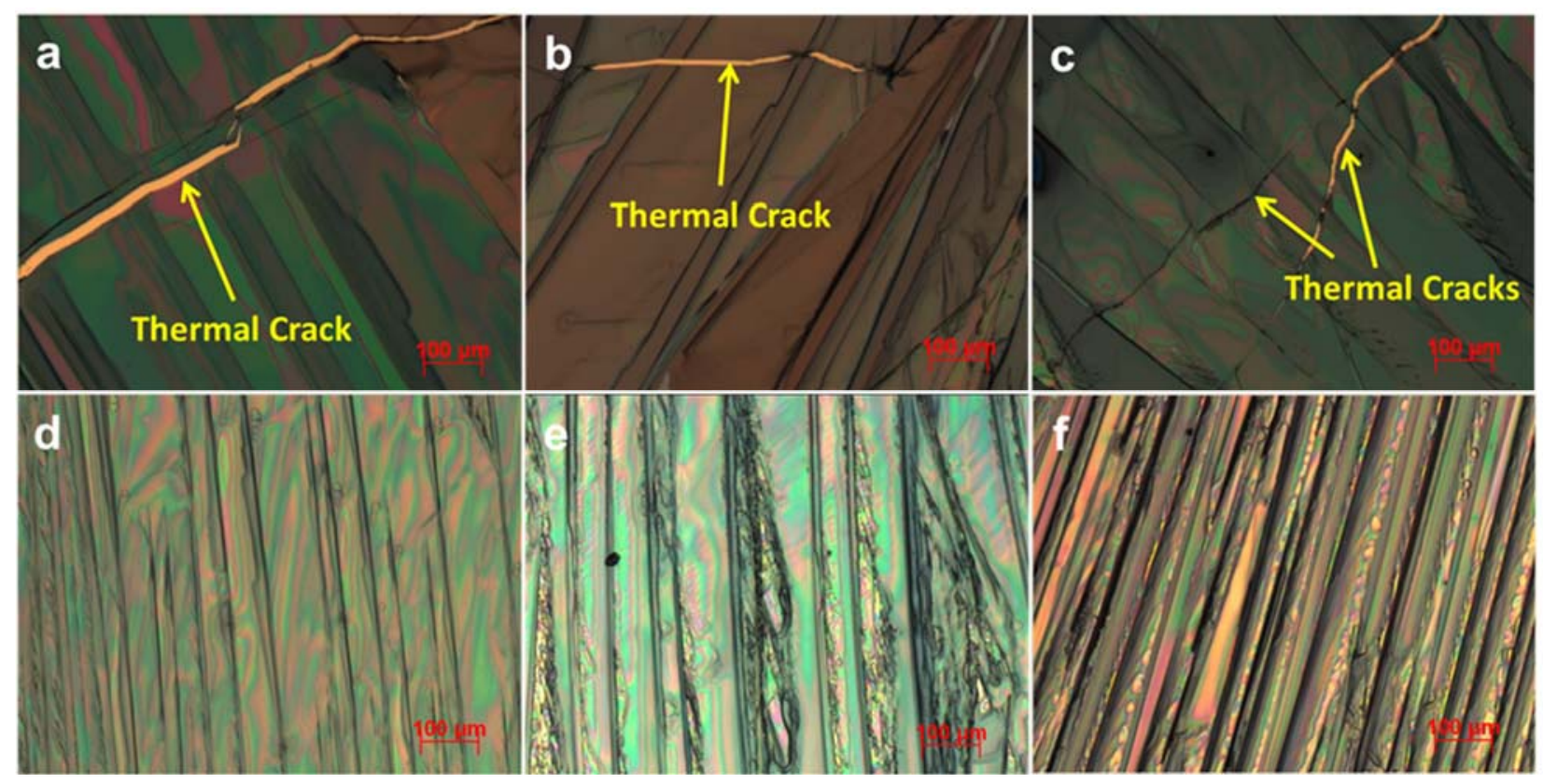

Figure 2. Polarized optical micrographs of (a-c) pure TIPS pentacene films showing large crystals with thermal cracks and (d-f) TIPS pentacene/P $\alpha$ MS blend films showing relatively small crystals without any cracks.

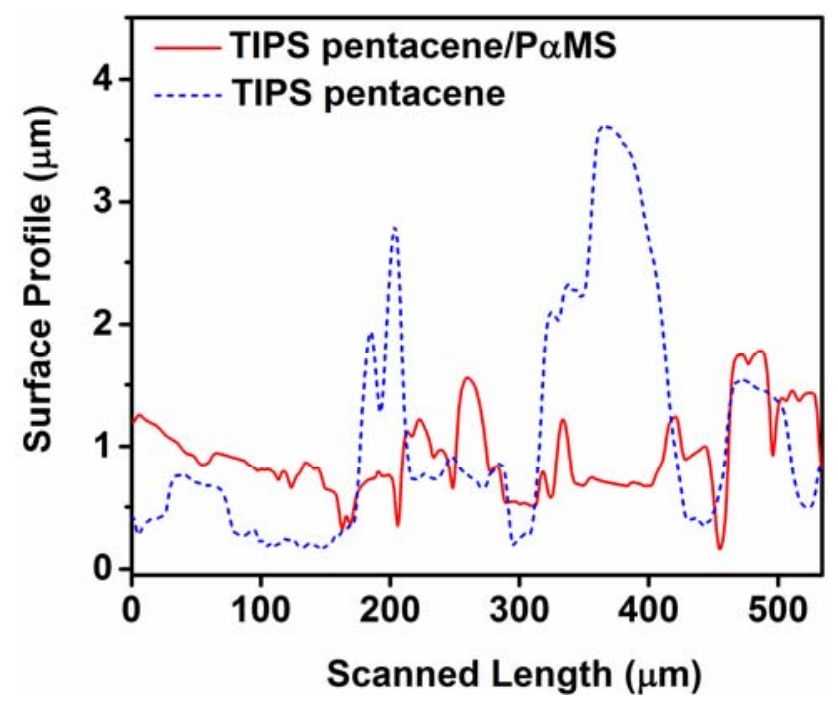

Figure 3. Comparison of film topography between TIPS pentacene/P $\alpha \mathrm{MS}$ film via temperature gradient technique and pure TIPS pentacene from simple drop casting. 

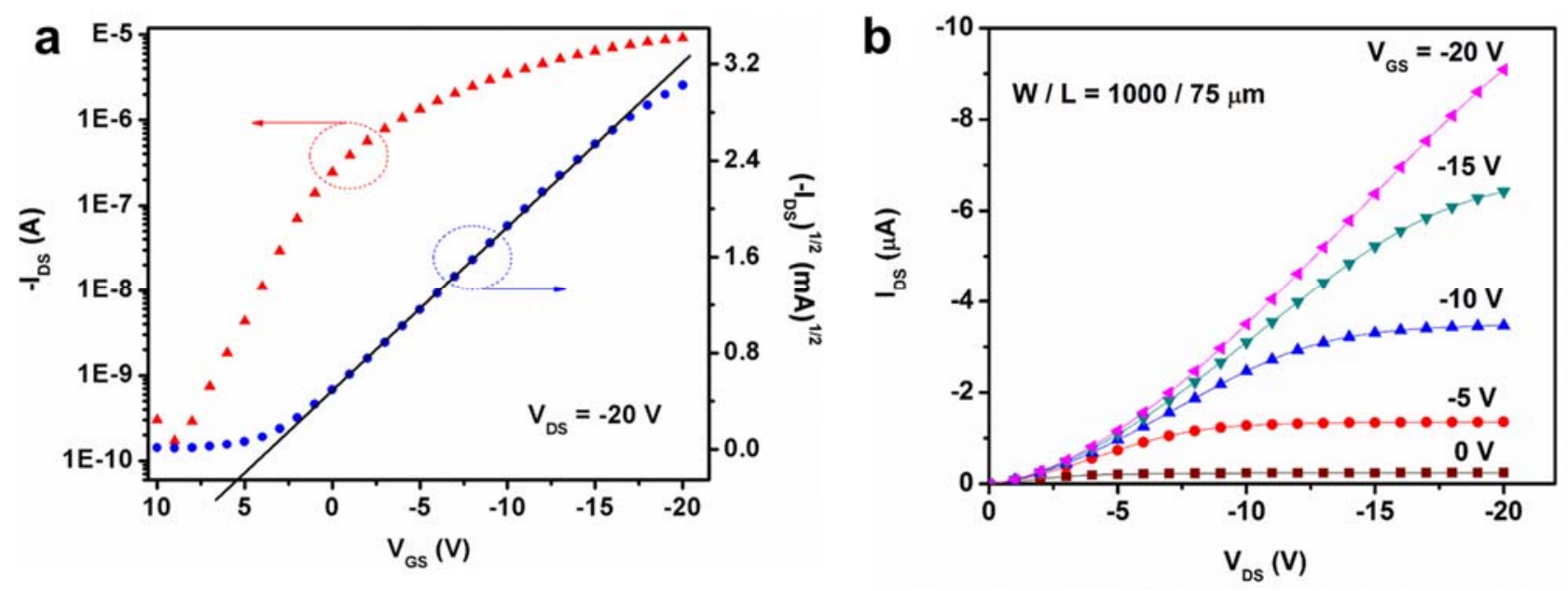

Figure 4. (a) Transfer and (b) output characteristics of bottom-gate, top-contact TIPS pentacene $/ \mathrm{P} \alpha \mathrm{MS}$ OTFTs from the optimal blend ratio of $2: 1$ at a $3.5^{\circ} \mathrm{C} / \mathrm{cm}$.

a
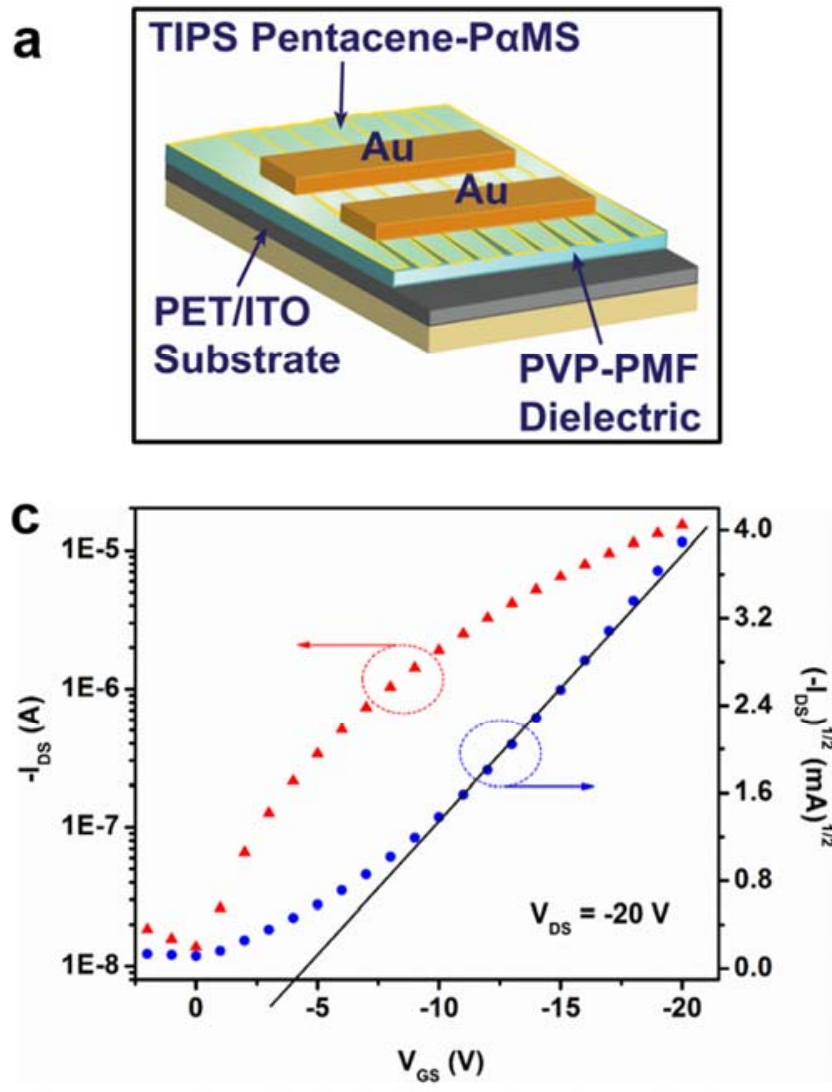

b
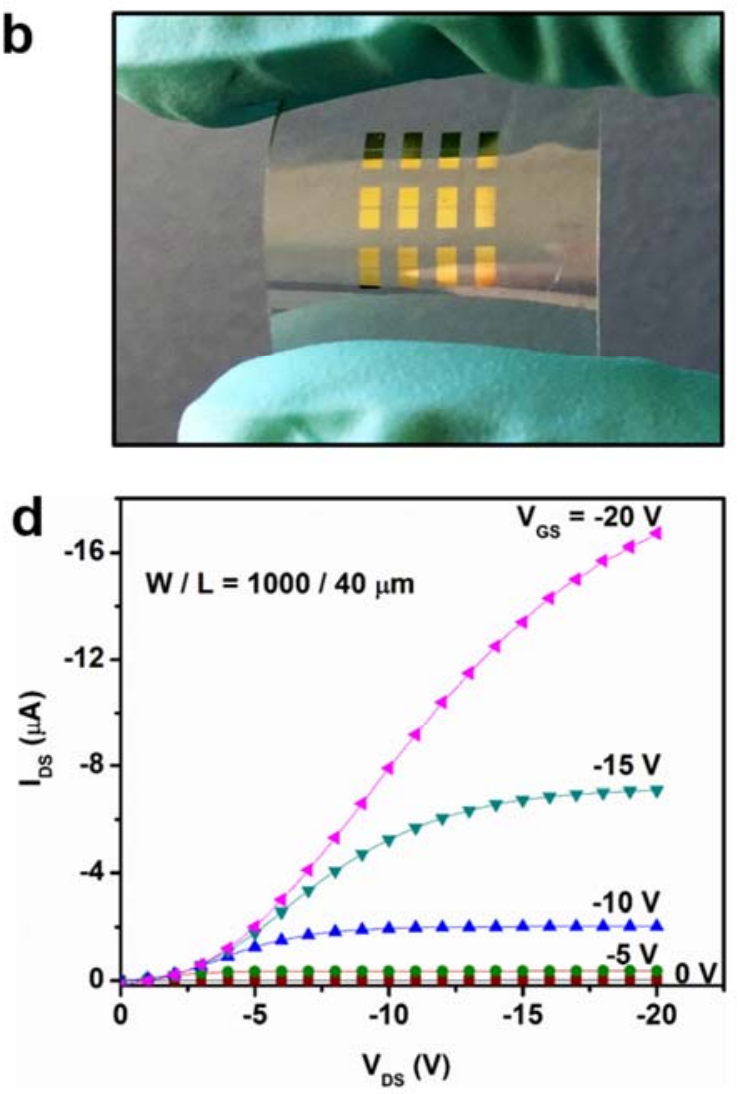
Figure 5. (a) Device configuration of bottom-gate, top-contact TIPS pentacene/P $\alpha$ MS OTFTs. (b) A digital picture that shows the OTFTs on ITO/PET flexible substrate. (c) Transfer and (d) output characteristics of TIPS pentacene/P $\alpha$ MS OTFTs on the ITO/PET flexible substrate.
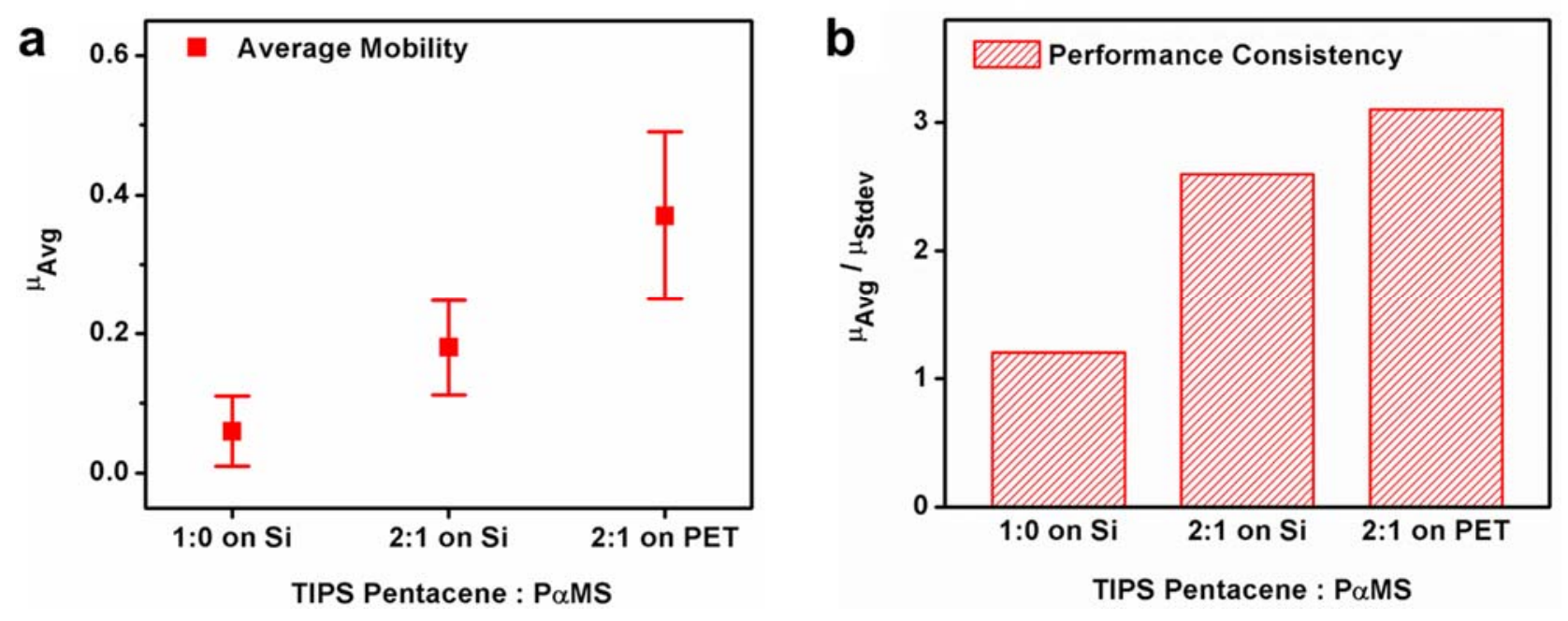

Figure 6. (a) Average hole mobility and (b) corresponding performance consistency of pure TIPS pentacene based OTFTs (1:0 blend ratio), and TIPS pentacene/P $\alpha$ MS based OTFTs (2:1 blend ratio) on silicon and ITO/PET substrates, respectively. 


\section{Reference}

[1] L. Yang, M. Tabachnyk, S.L. Bayliss, M.L. Bohm, K. Broch, N.C. Greenham, R.H. Friend, B. Ehrler, Solution-Processable Singlet Fission Photovoltaic Devices, Nano Lett., 15 (2015) 354-358.

[2] C.Y. Wong, B.L. Cotts, H. Wu, N.S. Ginsberg, Exciton dynamics reveal aggregates with intermolecular order at hidden interfaces in solution-cast organic semiconducting films, Nature Comm., 6 (2015) 7.

[3] Z.R. He, J.H. Chen, J.K. Keum, G. Szulczewski, D.W. Li, Improving performance of TIPS pentacenebased organic thin film transistors with small-molecule additives, Org. Electron., 15 (2014) 150155.

[4] W.H. Lee, J.A. Lim, D. Kwak, J.H. Cho, H.S. Lee, H.H. Choi, K. Cho, Semiconductor-Dielectric Blends: A Facile All Solution Route to Flexible All-Organic Transistors, Adv. Mater., 21 (2009) 4243-+.

[5] N. Shin, J. Kang, L.J. Richter, V.M. Prabhu, R.J. Kline, D.A. Fischer, D.M. DeLongchamp, M.F. Toney, S.K. Satija, D.J. Gundlach, B. Purushothaman, J.E. Anthony, D.Y. Yoon, Vertically Segregated Structure and Properties of Small Molecule-Polymer Blend Semiconductors for Organic Thin-Film Transistors, Adv. Func. Mater., 23 (2013) 366-376.

[6] S. Hunter, J.H. Chen, T.D. Anthopoulos, Microstructural Control of Charge Transport in Organic Blend Thin-Film Transistors, Adv. Func. Mater., 24 (2014) 5969-5976.

[7] Y.B. Yuan, G. Giri, A.L. Ayzner, A.P. Zoombelt, S.C.B. Mannsfeld, J.H. Chen, D. Nordlund, M.F. Toney, J.S. Huang, Z.N. Bao, Ultra-high mobility transparent organic thin film transistors grown by an off-centre spin-coating method, Nature Comm., 5 (2014) 4005.

[8] H. Minemawari, T. Yamada, T. Hasegawa, Crystalline film growth of TIPS-pentacene by doubleshot inkjet printing technique, Japan. J. Appl. Phys., 53 (2014) 05HC10.

[9] K. Kotsuki, H. Tanaka, S. Obata, S. Stauss, K. Terashima, K. Saiki, The importance of spinning speed in fabrication of spin-coated organic thin film transistors: Film morphology and field effect mobility, Appl. Phys. Lett., 104 (2014) 4.

[10] Z.R. He, N. Lopez, X.L. Chi, D.W. Li, Solution-based 5, 6, 11, 12-tetrachlorotetracene crystal growth for high-performance organic thin film transistors, Org. Electron., 22 (2015) 191.

[11] Z.R. He, S. Shaik, S. Bi, J.H. Chen, D.W. Li, Air-stable solution-processed n-channel organic thin film transistors with polymer-enhanced morphology, Appl. Phys. Lett., 106 (2015) 183301.

[12] J.H. Chen, M. Shao, K. Xiao, Z.R. He, D.W. Li, B.S. Lokitz, D.K. Hensley, S.M. Kilbey, J.E. Anthony, J.K. Keum, A.J. Rondinone, W.Y. Lee, S.Y. Hong, Z.A. Bao, Conjugated Polymer-Mediated Polymorphism of a High Performance, Small-Molecule Organic Semiconductor with Tuned Intermolecular Interactions, Enhanced Long-Range Order, and Charge Transport, Chem. Mater., 25 (2013) 4378-4386.

[13] Z.R. He, J.H. Chen, Z.Z. Sun, G. Szulczewski, D.W. Li, Air-flow navigated crystal growth for TIPS pentacene-based organic thin-film transistors, Org. Electron., 13 (2012) 1819-1826.

[14] Z.R. He, D.W. Li, D.K. Hensley, A.J. Rondinone, J.H. Chen, Switching phase separation mode by varying the hydrophobicity of polymer additives in solution-processed semiconducting smallmolecule/polymer blends, Appl. Phys. Lett., 103 (2013) 113301.

[15] S. Bi, Z.R. He, J.H. Chen, D.W. Li, Solution-grown small-molecule organic semiconductor with enhanced crystal alignment and areal coverage for organic thin film transistors, AIP Advances, 5 (2015) 077170.

[16] R. Hamilton, J. Smith, S. Ogier, M. Heeney, J.E. Anthony, I. McCulloch, J. Veres, D.D.C. Bradley, T.D. Anthopoulos, High-Performance Polymer-Small Molecule Blend Organic Transistors, Adv. Mater., 21 (2009) 1166-1171. 
[17] K. Asare-Yeboah, R.M. Frazier, G. Szulczewski, D. Li, Temperature gradient approach to grow large, preferentially oriented 6,13-bis(triisopropylsilylethynyl) pentacene crystals for organic thin film transistors, J. Vac. Sci. Tech. B, 32 (2014) 052401.

[18] J.H. Chen, C.K. Tee, J.Y. Yang, C. Shaw, M. Shtein, D.C. Martin, J. Anthony, Thermal and mechanical cracking in bis(triisopropylsilylethnyl)pentacene thin films (vol 44, pg 3631, 2006), J. Poly. Sci. Pt. B - Poly. Phys., 46 (2008) 1878-1878.

[19] F.C. Chen, C.S. Chuang, Y.S. Lin, L.J. Kung, T.H. Chen, H.P.D. Shieh, Low-voltage organic thin-film transistors with polymeric nanocomposite dielectrics, Org. Electron., 7 (2006) 435-439.

[20] D.K. Hwang, C. Fuentes-Hernandez, J.D. Berrigan, Y.N. Fang, J. Kim, W.J. Potscavage, H. Cheun, K.H. Sandhage, B. Kippelen, Solvent and polymer matrix effects on TIPS-pentacene/polymer blend organic field-effect transistors, Journal of Materials Chemistry, 22 (2012) 5531-5537.

[21] T. Ohe, M. Kuribayashi, R. Yasuda, A. Tsuboi, K. Nomoto, K. Satori, M. Itabashi, J. Kasahara, Solution-processed organic thin-film transistors with vertical nanophase separation, Appl. Phys. Lett., 93 (2008) 3.

[22] J. Kang, N. Shin, D.Y. Jang, V.M. Prabhu, D.Y. Yoon, Structure and properties of small moleculepolymer blend semiconductors for organic thin film transistors, J. Am. Chem. Soc., 130 (2008) 12273-12275.

[23] H.L. Zhong, J. Smith, S. Rossbauer, A.J.P. White, T.D. Anthopoulos, M. Heeney, Air-Stable and High-Mobility n-Channel Organic Transistors Based on Small-Molecule/Polymer Semiconducting Blends, Adv. Mater., 24 (2012) 3205-3211.

[24] S.Y. Cho, J.M. Ko, J.Y. Jung, J.Y. Lee, D.H. Choi, C. Lee, High-performance organic thin film transistors based on inkjet-printed polymer/TIPS pentacene blends, Org. Electron., 13 (2012) 1329-1339.

[25] Z.R. He, K. Xiao, W. Durant, D.K. Hensley, J.E. Anthony, K.L. Hong, S.M. Kilbey, J.H. Chen, D.W. Li, Enhanced Performance Consistency in Nanoparticle/TIPS Pentacene-Based Organic Thin Film Transistors, Adv. Func. Mater., 21 (2011) 3617-3623.

[26] M. B. Madec , D. Crouch , G. R. Llorente , T. J. Whittle , M. Geoghegan ,S. G. Yeates , J. Mater. Chem. $2008,18,3230$

[27] J. Kim, J. Jeong, H.D. Cho, C. Lee, S.O. Kim, S.K. Kwon, Y. Hong, All-solution-processed bottomgate organic thin-film transistor with improved subthreshold behaviour using functionalized pentacene active layer, J. Phys. D - Appl. Phys., 42 (2009) 6.

[28] C. Pitsalidis, N. Kalfagiannis, N.A. Hastas, P.G. Karagiannidis, C. Kapnopoulos, A. loakeimidis, S. Logothetidis, High performance transistors based on the controlled growth of triisopropylsilylethynyl-pentacene crystals via non-isotropic solvent evaporation, Rsc Advances, 4 (2014) 20804-20813. 


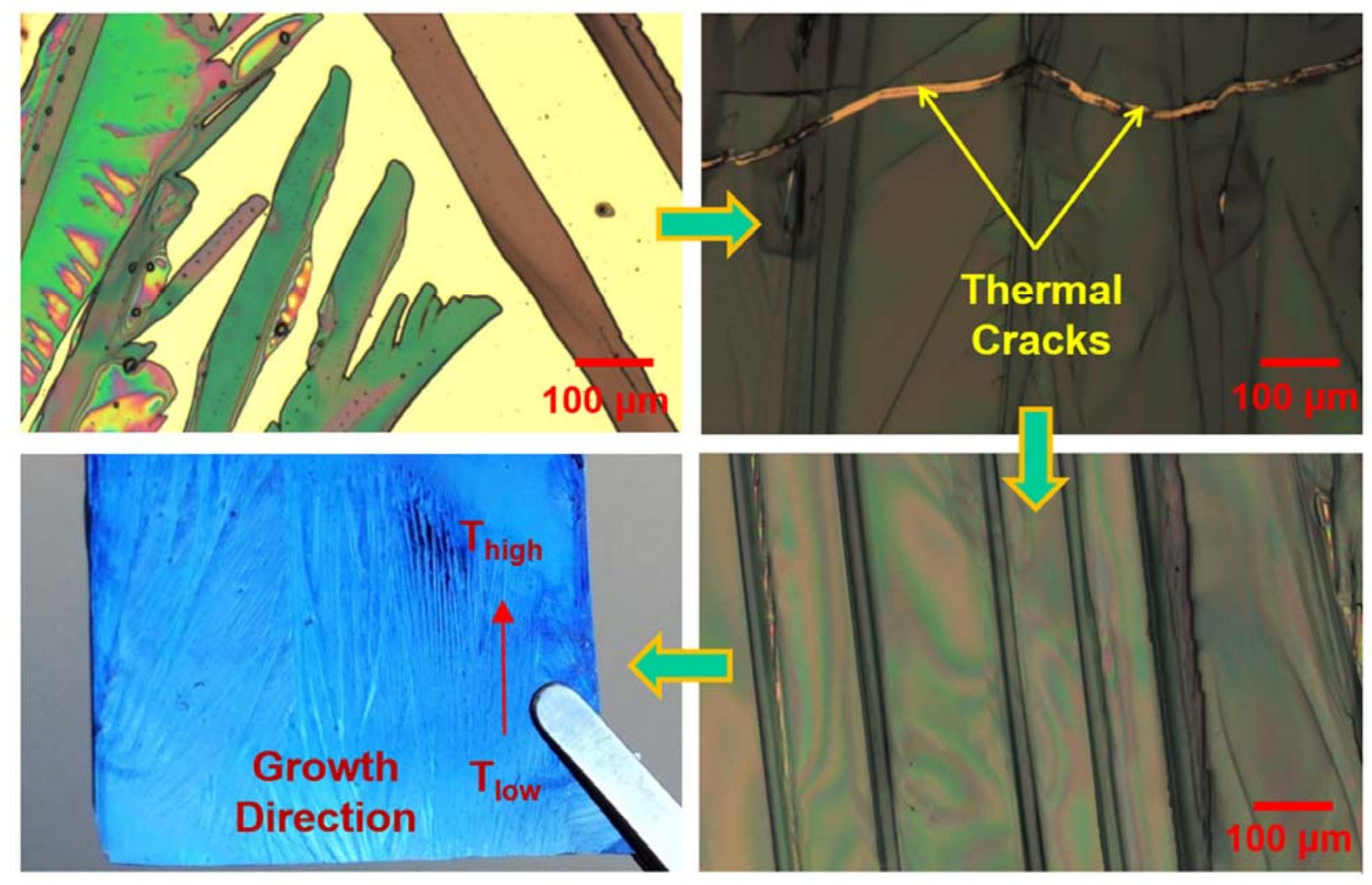

Graphical Abstract 